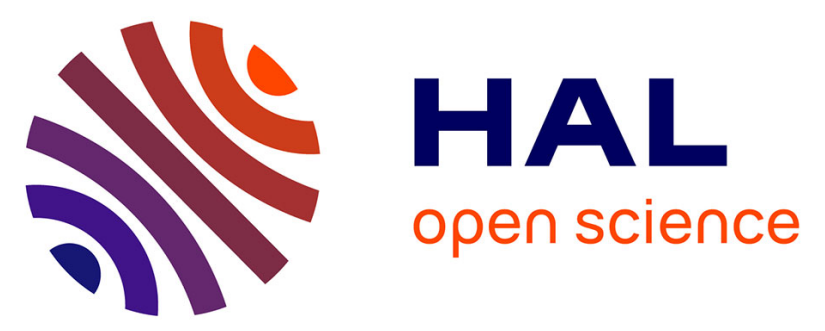

\title{
Diamond for actinide traces detection and spectrometry in liquids
}

\author{
Michal Pomorski, Christine Mer-Calfati, Jacques de Sanoit, Thuan Quang \\ Tran, P. Bergonzo
}

\section{To cite this version:}

Michal Pomorski, Christine Mer-Calfati, Jacques de Sanoit, Thuan Quang Tran, P. Bergonzo. Diamond for actinide traces detection and spectrometry in liquids. ANIMMA 2013 - 3rd International Conference on Advancements in Nuclear Instrumentation, Measurement Methods and their Applications, Jun 2013, Marseille, France. pp.6727986, 10.1109/ANIMMA.2013.6727986 cea-01822342

\section{HAL Id: cea-01822342 https://hal-cea.archives-ouvertes.fr/cea-01822342}

Submitted on 3 Feb 2022

HAL is a multi-disciplinary open access archive for the deposit and dissemination of scientific research documents, whether they are published or not. The documents may come from teaching and research institutions in France or abroad, or from public or private research centers.
L'archive ouverte pluridisciplinaire HAL, est destinée au dépôt et à la diffusion de documents scientifiques de niveau recherche, publiés ou non, émanant des établissements d'enseignement et de recherche français ou étrangers, des laboratoires publics ou privés. 


\title{
Diamond for Actinide Traces Detection and Spectrometry in Liquids
}

\author{
Michal Pomorski, Christine Mer, Jacques de Sanoit, Thuan Quang Tran, and Philippe Bergonzo
}

\begin{abstract}
We describe here a new approach for the detection and identification of actinides ( $\mathrm{Am}, \mathrm{Pu}, \mathrm{Cm}$ etc) at very low activity levels in aqueous solution. The measurement consists at first in the electroprecipitation of the actinides ions as insoluble hydroxides directly onto a boron doped nanocrystalline diamond (BNCD) electrode deposited on an $\alpha$-particle detector ( $\mathrm{Si}$ or Si-PIN diode), followed by $\alpha$-particles detection using frontend nuclear electronics. After $\alpha$-particles counting, spectrometry, the detector can be easily decontaminated using anodization in aqueous solution to be able to be reused at once. The detection limit of the described prototype system can be estimated as low as a few $\mathrm{mBq} / \mathrm{L}$ (for one day counting) to several $\mathrm{mBq} / \mathrm{L}$ for 5h counting and currently achieved energy resolution amounts to $\Delta E_{F W H M} / E_{\alpha}=2.3 \%$ for pulse height spectra of $5.486 \mathrm{MeV}$ $\alpha$-particles emitted by ${ }^{241} \mathrm{Am}$, measured directly in water.
\end{abstract}

Index Terms $-\alpha$ particle spectroscopy, actinides, electroprecipitation, CVD diamond, detection.

\section{INTRODUCTION}

$\mathbf{T}$ HE quality of the water we use every day is an essential governing parameter of the public health. In our societies, the preservation of the integrity of the drinking water supply is considered as a major stake. Numerous pollutants can affect more or less durably the quality of the water which we use every day. Besides classic contaminants as organic products, heavy metals, and bacteriological pollutants, the quality of the water can be also severely affected by radioactivity. The water network can be seen as vulnerable to attacks created by nuclear accidents or by terrorists attacks such as contamination through reservoirs, back-flow from domestic water supply taps, etc. The recent example of Fukushima alerted the world on the weakness of our modern societies to a radioactive contamination of the water supply chains for essential needs such as water, but also food, beverages etc. Equally, radionuclides coming from the human activity can result from the controlled emission of radioactive substances in the atmosphere or in the aquatic and marine media, supervised by proper authorities. For example the AREVA factory at La Hague France (the French nuclear fuel reprocessing plant) is authorised to release annually a total $\alpha$ emitters activity of $0.14 \mathrm{TBq}$ in the sea and $0.00001 \mathrm{TBq}$ in the atmosphere [1]. Among the multiple constituents of radioactive wastes, the actinide elements are of major importance due to their

All authors are with CEA, LIST, Diamond Sensors Laboratory, CE Saclay, 91191 Gif-sur-Yvette CEDEX - France (e-mails: michal.pomorski@cea.fr christine.mer@cea.fr, jacques.desanoit@cea.fr, quang.thuan.tran@cea.fr, philippe.bergonzo@cea.fr)

This work is performed in the framework of ActiFind project. The authors would like to thank ANR (French Research Agency) for the funding of this work. long half-lives of hundreds to hundred thousands of years, and their radiological and chemical toxicity for human health. The ability to directly measure the $\alpha$ activity of liquid media at trace levels constitutes a real breakthrough. Trace detection for radiation emitters is available from the measurement of beta or gamma emitters that are detected in shielded vessels. However, for $\alpha$ particles, since their penetration distance is confined down to a few tens of microns, no measurement can be made from the outside of the container, nor from the use of immersed detectors that will only probe a weak liquid thickness of typically $2 \mu \mathrm{L} / \mathrm{cm}^{2}$ [2], [3], [4]. As a result, due to the self-absorption of $\alpha$ particles in water, conventional methods consist of evaporating the medium to obtain a solid condensate on which conventional $\alpha$ spectroscopy can be used [5]. This approach is compatible with very clean water, but cannot be used with contaminated waters with any form of precipitate, waste water, urine, juice etc as the solid residue will also result in self-absorbing the $\alpha$ activity and lead to underestimated evaluations. Here therefore the main objective of this paper is the realization of a high sensitivity $\alpha$-particle detection system working in water for the detection of dissolved actinides. So far, no system exists that is enabling the measurement of radioactive water in a portable, easy to handle system that can be established for early stage detection of nuclear attacks or accidents, as well as for permanent water quality monitoring. In the following, we will introduce an innovative approach taking advantage of synthetic diamond properties, that will be of high benefit for sensitive detection.

\section{EXPERIMENTAL AND METHODS}

\section{A. Fabrication of BNCD/Si and BNCD/SiPIN Sensors}

Two generations of sensors were produced for the purpose of measuring the actinides traces in liquid phase with the electrochemically assisted pre-concentration step. (i) The first generation so-called: counter-grade boron doped nanocrystalline diamond (CG-BNCD/Si) sensors; allows counting of $\alpha$-particles emitted by decaying actinides atoms with clear separation of induced signals from the electronic noise and $\alpha$-particle detection efficiency close to $100 \%$ (in $2 \pi$ geometry). (ii) An improved second generation sensors so called spectroscopic-grade (SG-BNCD/SiPIN), allows both actinides counting and identification through the $\alpha$-particles energy loss spectroscopy. For the (i) CG-BNCD/Si sensors fabrication, a thin $(200 \mu \mathrm{m})$ high resistivity $\left(10^{4} \Omega \mathrm{cm}\right)$ intrinsic silicon wafer whether in the case of (ii) spectroscopic-grade sensors, commercial SiPIN diodes were used as a substrate for BNCD thinelectrode chemical vapor deposition (CVD). In a first step the 


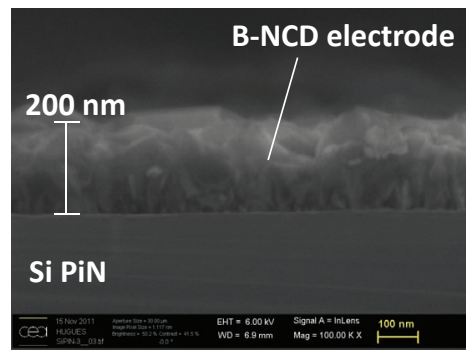

Fig. 1. A SEM photograph showing the cross-section of a $200 \mathrm{~nm}$ thick electrochemistry grade BNCD electrode deposited on a SiPIN substrate.

original diode metallization is stripped-off using hot aqua-regia $\left(\mathrm{HCl}: \mathrm{HNO}_{3}\right.$ - 3:1) solution. The bare substrate is then rinsed in DI water and dried. In the second step the P+ surface of the diode (or one surface of Si wafer) is seeded with aqueous solution of diamond nanoparticles using spin-coating method. Such prepared sample is loaded into a microwave-plasma assisted CVD reactor. We used trimethyloboron (TMB) as a dopant source to obtain highly boron doped $\left(10^{21}[\mathrm{~B}] / \mathrm{cm}^{3}\right)$ diamond films [6]. The CVD growth parameters were following; MW power: $1.8 \mathrm{~kW}$, substrate temperature: $1025 \mathrm{~K}, \mathrm{H}_{2}$ flow: 88 sscm, $\mathrm{CH}_{4}$ flow: $0.22 \mathrm{sscm}, \mathrm{TMB}$ (2000 ppm): $12 \mathrm{sscm}$.

One must note that the BNCD electrode corresponds to the dead detector volume not contributing to the signal formation, thus it must be kept as thin as possible, in current work, 200 $500 \mathrm{~nm}$ thick BNCD layers were synthetized, however it could be further thinned down (50-100 nm pin-holes free BNCD films seems feasible). Figure 1 displays a SEM micrograph with a cross-sectional view of a $200 \mathrm{~nm}$ thick BNCD layer deposited onto the SiPIN substrate. After diamond growth, the central area of about $0.8 \mathrm{~cm}^{2}$ of the device is masked with $200 \mathrm{~nm}$ aluminium sputtered mask and in the following step, peripheral regions of BNCD diamond are etched using Ar/O plasma. Later the aluminium mask is stripped-off with an aqua-regia solution. The back surface of the diode (or high resistivity silicon) is coated with $200 \mathrm{~nm}$ of gold, forming an ohmic back contact. The device is mounted onto an insulating support and contacted to a coaxial cable using silver loaded epoxy resin. We use a highly resistive and electrochemically inactive epoxy resin to encapsulate the device leaving only a BNCD diamond entrance electrode with a direct contact to the analyzed solution. The electrochemically active BNCD area was estimated from its photograph by integrating the sensor surface using an image processing software and amounts to about $0.4 \mathrm{~cm}^{2}$ for each sensor. Prior to the measurements in radioactive solutions the properties of each BNCD electrode were evaluated using standard electrochemical techniques. For instance, Figures 2 and 3 show the electrochemical impedance spectroscopy diagrams (Nyquist plot) and cyclic voltammetry curves of a CG-BNCD/Si sensor, respectively. The measurements are performed using an equimolar solution of potassium ferro-ferricyanide $10^{-3} \mathrm{M}$ (outer sphere redox couple) with $\mathrm{KCl}$ as supporting electrolyte. The figures demonstrate the very high reactivity of the electrodes with high electron

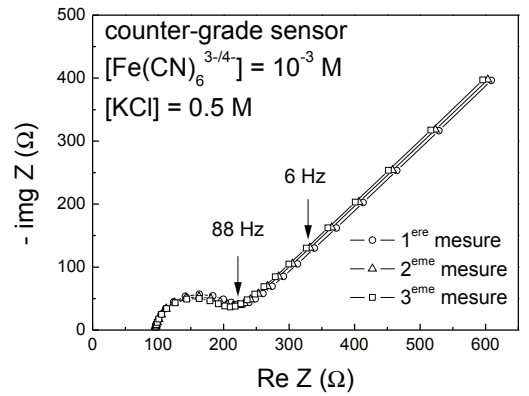

Fig. 2. Electrochemical impedance spectroscopy plot (Nyquist plot) of a CG$\mathrm{BNCD} / \mathrm{Si}$ sensor showing the series resistance value (first intercept $100 \Omega$ ) and the electron transfer resistance (derived from the second intercept 125 $\Omega)$.

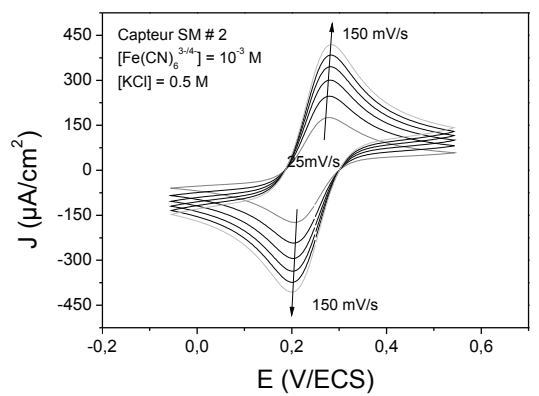

Fig. 3. Cyclic voltammetry curves performed with the CG-BNCD/Si sensor showing the electrochemical activity of the BNCD layer. The measurements are performed using an equimolar solution of potassium ferro-ferricyanide $10^{-} 3 \mathrm{M}$ (outer sphere redox couple) with $\mathrm{KCl}$ as supporting electrolyte.

transfer rate values and their remarkable reproducibility.

\section{B. Electro-precipitation of Actinides on BNCD Entrance Elec- trode}

Simple electrochemistry cannot by itself suffice to make a metallic layer of actinides on an electrode surface from an aqueous solution. All of the values of the standard potentials $\mathrm{E}_{0}$ of various couples $\mathrm{M}^{3+} / \mathrm{M}^{0}$ (with $\mathrm{M}=\mathrm{Am}, \mathrm{Cm}, \mathrm{Pu}$ ) are very negative $\left(\mathrm{E}_{0}<-2 \mathrm{~V} / \mathrm{ENH}\right)$ [7]. In addition, in acid aqueous solutions, the field of potentials is limited on the reduction side to: $\mathrm{E}=0 \mathrm{~V} / \mathrm{ENH}$ through system $\mathrm{H}_{2} / \mathrm{H}^{+}$ and on the oxydation side to: $\mathrm{E}=+1.23 \mathrm{~V} / \mathrm{ENH}$ through system $\mathrm{H}_{2} \mathrm{O} / \mathrm{O}_{2}$. Therefore, no electrochemical activity takes place besides the electrochemical decomposition of the solvent (reduction/oxidation of water). The whole of the current fed through the electrode being used by these reactions building what the electrochemists call the solvent wall. One can overcome this experimental difficulty by taking into account that actinide ions are very sensitive to hydrolysis, which is a particular case of the complexation reactions. By increasing the $\mathrm{pH}$ of an aqueous solution containing actinide ions, the structure of ions is modified: protons become expelled by water hydration molecules towards non-binding water, the actinide ion accumulate hydroxyl ions $\left(\mathrm{OH}^{-}\right)$in the internal sphere and form hydroxo mono or polynuclear complexes to 
form solid hydroxides in the form of $\mathrm{M}(\mathrm{OH})_{3}$. As a result, ion hydrolysis of actinides will not take place as long as they are kept in acidic solutions at concentrations above $1 \mathrm{M}$ or/and by the addition of complexing ions $\left(\mathrm{NO}^{3-}, \mathrm{SO}_{4}^{2-}\right)$ which will delay the aforementioned hydrolysis reactions. It is easy to locally modify the $\mathrm{pH}$ of the surface of the working electrode by water reduction, that results in the formation of a basic layer $(\mathrm{pH} \mathrm{12-13)}$ according to the following reaction (1):

$$
\mathrm{H}_{2} \mathrm{O}+\mathrm{H}^{+}+2 e \rightarrow \mathrm{OH}^{-}+\mathrm{H}_{2}
$$

The electro precipitation of insoluble actinide hydroxide can thus be carried out even in slightly acidic solution because of the formation of this basic layer next to the working electrode according to the reaction (2):

$$
\mathrm{M}^{3+}+3 \mathrm{OH}^{-} \rightarrow \mathrm{M}(\mathrm{OH})_{3}
$$

When the reduction current is switched off, the basic layer disappears and the actinide hydroxide can dissolve more or less quickly in the liquid medium, thus the solution must be basified just before swithing off the current. Please note that this process is not electrodeposition, which is a term used for the electrochemical reduction of ions in a metallic state. Here, electro precipitation from which the product does not result from a transfer of electrons between the electrode and a metallic cation takes place. This method was initiated by Talvitie for stainlesssteel substrates in 1972 [8] then modernized successively in 1996 by Becceril [9] and in 2000 by Tsoupko-Sitnikov [10]. To electroprcipitate the actinides ions on the BNCD sensor entrance electrode, the aqueous medium containing actinides to be analyzed is chemically adjusted in terms of both $\mathrm{pH}$ and salt concentration (using $\mathrm{Na}_{2} \mathrm{SO}_{4}$ ). The adjustment of the $\mathrm{pH}$ to a value in the range of 3 and 4.5 is necessary, as well as that of the basic complexing salt to a concentration of $0.3 \mathrm{M}$ to allow the control of both the hydrolysis of actinide ions and their complexation. The charge of added salt thus provides good conductivity of the electrolyte reducing the voltage drop across the electrolytic cell. The electro precipitation of actinide ions on the surface of boron doped diamond (cathode) is made by connecting a negative bias to the sensor and the positive one to the anode; here made from a platinum wire. The operating mode is the galvanostatic mode at low current densities $\left(-1.5<J<-6 \mathrm{~mA} / \mathrm{cm}^{2}\right)$ and vigorous stirring $\left(10^{3} \mathrm{rpm}\right)$ for a period of 120 minutes. The electro-precipitation experimental arrangement is illustrated in Figure 4

\section{C. $\alpha$ Particle Detection}

The second step consists of the measurement of the electroprecipitated actinide ions on the surface of the sensor BNCD entrance electrode. For this purpose the sensor is kept in the solution, but placed in a metalic lightight container and connected to a spectroscopic chain as depicted in Figure 5. In the present experiment a custom made fast charge sensitive amplifier FCSA [11] was used to amplify and to shape the $\alpha$-particles induced current signals, which is digitalized and registered as pulse-height spectra on a digital-storage oscilloscope (DSO). Nevertheless, any classical/commercial $\alpha$

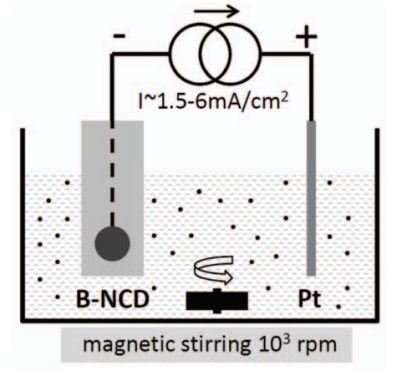

Fig. 4. The experimental arrangement used for electro precipitation of actinides ions onto the CG-BNCD/Si and SG-BNCD/SiPIN sensors

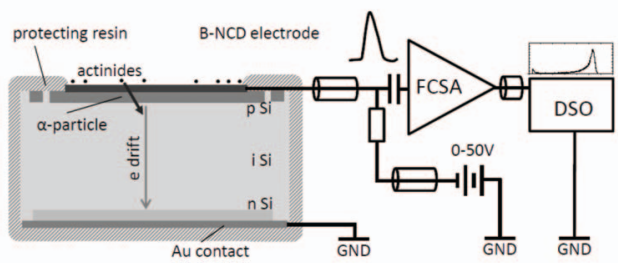

Fig. 5. A schematic view of the SG-BNCD/SiPIN sensor and associated signal processing electronics

spectrometry chain designed to work with silicon detectors can also efficiently be used to probe the signal from our sensors. The $\alpha$-particles from the precipitated actinide layer on the diamond film will interact in the silicon ionization chamber inducing current pulses. The number of counts recorded by the detector is proportional (in the best case about $50 \%$ of decaying nuclides can be detected, due to the $2 \pi$ geometry) to the amount of $\alpha$ species present at the diamond surface, and the signal amplitude to the characteristic $\alpha$-particles energy allowing actinides identification. As a result, it comes very accurate to probe the actinide concentration and its origin in the solution thanks to a prior calibration of the device.

\section{RESUlTS AND Discussion}

\section{A. Sensors Pre-characterization with a Solid Source}

Directly after the fabrication and prior to the measurements with radioactive solutions the prototypes were evaluated in vacuum $\left(10^{-3}\right.$ mbar $)$ under irradiation of $5.48 \mathrm{MeV} \alpha$ particles from a $4 \mathrm{kBq}$ solid ${ }^{241} \mathrm{Am}$ source. The source of a diameter larger than that of the active area of the sensors (about $0.4 \mathrm{~cm}^{2}$ ) was placed at about $1 \mathrm{~mm}$ away from the BNCD entrance electrode, no collimator was used. In Figure 6 the comparison of the performances of three sensors is presented.

The light-grey curve in Figure 6 is the $\alpha$-particles pulseheight spectrum collected with a $\mathrm{CG}-\mathrm{BNCD} / \mathrm{Si}$ sensor operated at zero bias (sensor biasing was in fact not possible on this device due to high leakage current). Here, only a part of the induced charge can be collected due to the partially depleted silicon bulk. The charge carriers drift-diffusion is mainly governed by the space-charge region formed at the vicinity of the highly boron doped diamond layer and intrinsic Si bulk and only about $72 \%$ of e-h pairs are collected with a poor relative energy resolution of $\Delta E_{C G}=10 \%$. This value was obtained from Gaussian fit to the curve and defined as 


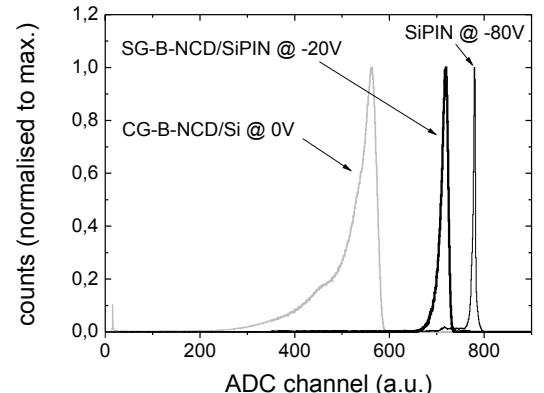

Fig. 6. Comparison of the performance of a CG-BNCD/Si, a SGBNCD/SiPIN sensors and a commercial SiPIN diode for $\alpha$-particle detection from an Am-241 spectroscopic-grade solid source Measurements were performed in vacuum at $10^{-3}$ mbar, spectra are normalized to the maximum number of counts.

$\Delta E=F W H M / X_{c}$, where FWHM is full width at half maximum of the peak and $X_{c}$ is the gauss centroid. Thickblack curve is a pulse height spectrum of a SG-BNCD/ SiPIN sensor operated at $-20 \mathrm{~V}$ and exhibiting a CCE of $93 \%$ and an improved energy resolution of $\Delta E_{S G}=2.4 \%$. Here the non-complete charge collection and limited energy resolution can be attributed to the dead BNCD entrance electrode layer thickness of about $400 \mathrm{~nm}$. This was confirmed by numerical simulation of $5.48 \mathrm{MeV} \alpha$-particles straggling in the detector dead layer using SRIM package [12]: for the $200-500 \mathrm{~nm}$ thick BNCD (with C-12 atoms and density $3.52 \mathrm{~g} / \mathrm{cm}^{2}$ ) dead layer simulations show an energy resolution $\Delta E_{S R I M}$ in the range of $1.4 \%(200 \mathrm{~nm})$ to $2.9 \%(500 \mathrm{~nm})$. Those results demonstrate that such SG-BNCD/SiPIN sensor response remains within the range of intrinsic energy resolution limitation. In Figure 6, the last curve compares both sensor responses to a reference non-modified commercial SiPIN detector (thin-black curve), exhibiting a $100 \%$ charge collection efficiency (CCE) and $\Delta E_{P i N}=0.6 \%$.

\section{B. Counter Grade Sensor Detection Performance}

Preliminary tests of the CG-BNCD/Si device were performed with a radioactive solution of ${ }^{241} \mathrm{Am}$ prepared from a $1 \mathrm{MBq}$ radioactive standard at the Laboratoire National Henri Becquerel (LNHB, CEA-Saclay). By diluting the standard in DI water, six daughter $100 \mathrm{ml}$ solutions of increasing activities were prepared within the activity range of 0.6 to $29 \mathrm{~Bq}$. The total $\alpha$ activity of each daughter solution was crosscalibrated using a Liquid Scintillation Technique (LST) at the same laboratory [13]. The precise activities values including measurement uncertainties are listed in Figure 7. Starting from the lowest activity, after 120 min of electro precipitation, we performed a 3 to $5 \mathrm{~h} \alpha$-particles counting for each daughter solution. After each step the sensor was decontaminated (see subsection D) and re-used in a daughter solution of higher activity. Figure 7 displays pulse height spectra acquired with a CG-BNCD/Si sensor.

By plotting the CG-BNCD/Si measured $\alpha$-particles count rate (in cps counts per second) derived from spectra integrals vs. total solution activity (from LST), clearly the linearity $\left(\mathrm{R}^{2}=0.998\right)$ of the system is evidenced in Figure 8 .

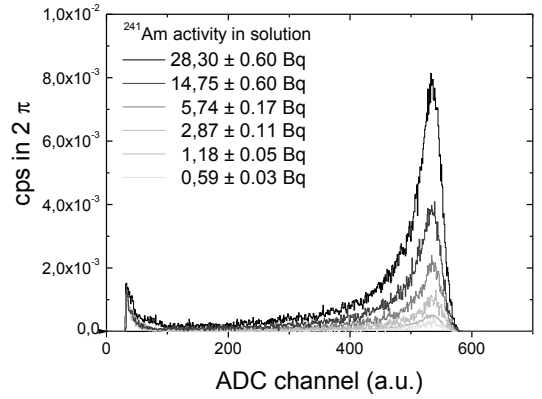

Fig. 7. Pulse-height spectra for varying concentrations of ${ }^{241} \mathrm{Am}$ in a $100 \mathrm{ml}$ water solution measured with a CG-BNCD/Si sensor. Total activities of the solution were calibrated with a liquid scintillation technique and are indicated in the graph.

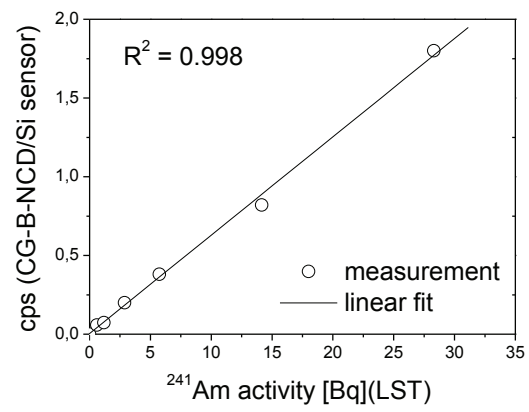

Fig. 8. Integrals of curves derived from Fig 7 vs. total ${ }^{241}$ Am solution activity from liquid scintillation technique. This graph shows the perfect linearity of the detection system

\section{Spectrosocopic Grade Sensor Detection Performance}

The spectroscopic capabilities of a SG-BNCD/SiPIN sensor were assessed using a solution of ${ }^{239} \mathrm{Pu}\left(\mathrm{E}_{\alpha}=5.39 \mathrm{MeV}\right)$ ${ }^{241} \mathrm{Am}\left(\mathrm{E}_{\alpha}=5.486 \mathrm{MeV}\right)$ and ${ }^{244} \mathrm{Cm}\left(\mathrm{E}_{\alpha}=5.86 \mathrm{MeV}\right)$. As in the previous subsection a $15 \mathrm{~Bq}(5 \mathrm{~Bq}$ of $\mathrm{Pu}, 5 \mathrm{~Bq}$ of $\mathrm{Am}$ and 5 $\mathrm{Bq}$ of $\mathrm{Cm}$ ) in $15 \mathrm{ml}$ mixed nuclide solution was prepared using LNHB radioactive standards. No LST cross-calibration was carried out, since precise knowledge of the solution activity was not important. Measured mixed-nuclide spectrum after electro precipitation step and $24 \mathrm{~h}$ counting is displayed in Figure 9.

Clearly, three peaks corresponding to the ${ }^{239} \mathrm{Pu},{ }^{241} \mathrm{Am}$ and ${ }^{244} \mathrm{Cm}$ emitted $\alpha$-particles can be distinguished in the pulse height spectrum, with ${ }^{239} \mathrm{Pu} \Delta E_{S G l q}=3.4 \%{ }^{241} \mathrm{Am} \Delta E_{S G l q}=$ $2.8 \%,{ }^{244} \mathrm{Cm} \Delta E_{S G l q}=2.3 \%$ respectively. The measured ${ }^{241} \mathrm{Am} \Delta E_{S G l q}$ is slightly higher compared to the measured one $\Delta E_{S G s d}(2.4 \%)$ with the ${ }^{241} \mathrm{Am}$ solid source and we tentatively attribute this to the observed degradation of SiPIN bulk resistivity. This sensor could not be operated at bias value of $-20 \mathrm{~V}$ thus having, partially depleted bulk. It is not clear to us what can be the cause of the degradation at the current state of investigation. One can suspect diffusion of impurities into not passivated Si bulk in areas not covered by BNCD electrode. Also low energy tails of the peaks raisng from $\alpha$-partciles stragling in diamond layer degrade slighlty the resolution of the peaks located at lower energy side of the spectrum. Finally 


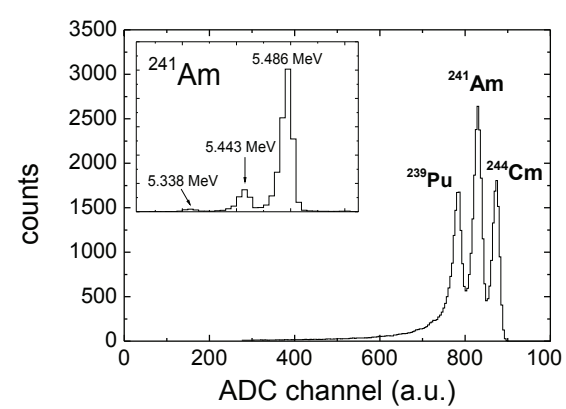

Fig. 9. An $\alpha$-partcile spectrum of mixed nuclide solution ( $\mathrm{Pu}, \mathrm{Am}, \mathrm{Cm})$ measured directly in liquid using electrochemically assisted SG-BNCD/Si sensor. Three peaks corresponding to $\alpha$-particles emitted by three actinides can be clearly distinguihed. The inset shows $\alpha$-particle spectrum of ${ }^{241} \mathrm{Am}$ deposited on a BNCD electrode as measured with an extrnal commercial Si $\mathrm{PiN}$ detector in vacuum $\left(10^{-3} \mathrm{mbar}\right)$ - Additinal two satelites lines are visible around main peak $(5.486 \mathrm{MeV})$ corresponding to alternative decay modes - a proof of spectroscopic grade precipitation of actinides on the sensors

in order to check if the quality of deposited actindes' film on the BNCD electrode is not a limiting factor for spectroscopy. A measurement with an external SiPIN detector was performed in vacuum $\left(10^{-3}\right.$ mbar $)$. Result for $\alpha$-particles emitted by ${ }^{241} \mathrm{Am}$ is displayed in the inset of Figure 9. Clearly three energies coresponding to different channels of $\alpha$ decay for ${ }^{241} \mathrm{Am}$ could be distinguished with $\Delta \mathrm{E}_{\text {ext }}=0.4 \%$ (value close to the state-of-the art $\alpha$-partciles spectroscopy systems) - a proof of spectroscopic grade electroprecipitation of actnides onto diamond film.

\section{Decontamination of the Sensors}

After each measurement, a light anodisation of the BNCD electrodes $\left(+6 \mathrm{~mA} / \mathrm{cm}^{2}\right.$ for 10 minutes $)$ simply results in the release of all pre-concentrated actinides species in the solution. This enables to clean the active surface layer of the devices prior to each following measurement. Performing a series of experiments, we achieved an avaregare decontamination efficiency of $99.98 \%$ for majority of the sensors. By extension, this technique can also lead to the fabrication of efficient electrochemical decontamination systems when large area BNCD electrodes are used. The media can be modified to a waste solution before releasing the radioactive traces in a dedicated waste water sample. Those devices can be therefore promoted to decontaminating systems when scaled up to several batches of large area BNCD electrodes.

\section{E. Long Term Performance of the Sensors}

In order to test long term stability of the sensors we performed a series of 30 cycles of electroprecipitationdecontamination on a SG-BNCD/Si sensor. Figure 10 displays two aquired spectra of ${ }^{241} \mathrm{Am}$ emitted $\alpha$-partciles for first and thirtieth experiments. Hardly any difference could be seen in both position and shape of the pulse-heights, also precipitationdetection efficiency did not change within the number of performed cycles.

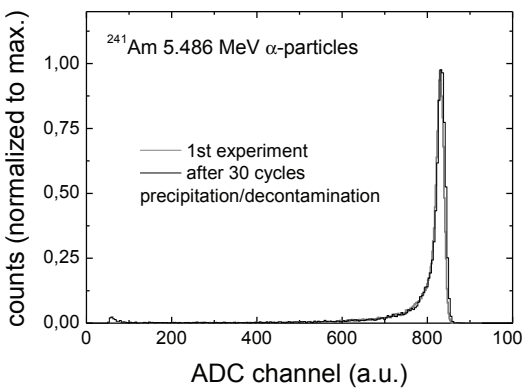

Fig. 10. Spectra of $\alpha$-particles emitted by ${ }^{241} \mathrm{Am}$ precipated on a SGBNCD/SiPIN sensor: for first experiment (light gray) and after 30 consecutive cycles of electroprecipitation-decontamination. We do not observe any degardation in: precipitation-detection efficiency, position and shape of the spectrum.

\section{CONCLUSION}

It is possible to directly perform $\alpha$-particles counting and energy loss spectroscopy in liquids using electrochemically assisted boron doped diamond/silicon sensors. The preconcentration step allows electro precipitation of actinides atoms $\left({ }^{239} \mathrm{Pu},{ }^{241} \mathrm{Am},{ }^{244} \mathrm{Cm}\right.$ tested in this work) directly onto the BNCD sensor entrance electrode. The obtained average precipitation efficiency of $13 \%$ (for $100 \mathrm{ml}$ of solution and 0.4 $\mathrm{cm}^{2}$ active area of the sensor) in presented experimets is still low compared to efficiencies matching up to $100 \%$ reported for stainless-steel or platinum substrates [7-9], thus a parallel work was started, focused on the improvement of this aspect; by adjusting the deposition parameters of the boron doped diamond electrode we were able to increase the efficiency close to $80 \%$ [14]. One other important aspect not addressed in this work is the influence of subsequent interfering metal ions (eg. Fe), which may tend to further reduce the efficiency due to competitive electrodeposition process. Nevertheless, we demonstrated capability of the first prototypes the possibility to detect traces of ${ }^{241} \mathrm{Am}$ at measured concentration levels as low as $0.5 \mathrm{~Bq} / 100 \mathrm{ml}$ as well as, actinides identification in mixed nuclide solution $\left({ }^{239} \mathrm{Pu},{ }^{241} \mathrm{Am},{ }^{244} \mathrm{Cm}\right)$ with relative energy resolution within the range of $\Delta \mathrm{E}_{F W H M}=3.4-2.3 \%$. Using a simple approximation, assuming a $1 \mathrm{~cm}^{2} \mathrm{CG}-\mathrm{BNCD} / \mathrm{Si}$ sensor, $24 \mathrm{~h}$ counting interval and measured electro precipitation efficiency, the detection limit (eg. statement: solution contaminated with actinides) for current prototypes can be reduced as low as a few $\mathrm{mBq} / \mathrm{L}$. By further development of the sensors' fabrication procedure eg. growing thinner BNCD films at low temperature we expect also improvement of the enregy resolution within the range of $\Delta \mathrm{E}_{F W H M}=1-0.5 \%$. This approach has been patented in 2010 and is now available for further development [15].

\section{ACKNOWLEDGMENT}

The authors would like to thank ANR (French Research Agency) for the funding of this work and collegues Sylvie Pierre and Philippe Cassette from Laboratoire National Henri Becquerel (LNHB, CEA-Saclay) for their help in metrological measurements. 


\section{REFERENCES}

[1] Journal officiel de la Rpublique franaise, n. 8, pp. 532,10 January 2007, http://www.legifrance.gouv.fr

[2] S.F. Kozlov, E.A. Konorova, M.I. Krapivin, V.A. Nadein, Usage of diamond detectors as immersed $\alpha$-counters, IEEE Transactions on nuclear science, Vol NS-24, N1 (1977) 242-243.

[3] P. Bergonzo, F. Foulon, A. Brambilla, D. Tromson, C. Jany, S. Haan, Corrosion hard CVD diamond $\alpha$ particle detectors for nuclear liquid source monitoring, Diamond and Related Materials. Vol 9 (2000) 10031007.

[4] O.B. Egorov, R.S. Addleman, M.J. OHara, T. Marks, J.W. Grate, Direct measurement of $\alpha$ emitters in liquids using passivated ion implanted planar silicon (PIPS) diode detectors, Nuclear Instruments and Methods in Physics Research A 537 (2005) 600-609.

[5] Semkow TM, Khan AJ, Haines DK, Bari A., Rapid $\alpha$ spectroscopy of evaporated liquid residues for emergency response, Health Phys. 2009 Apr;96(4):432-41

[6] E. Vanhove, J. de Sanoit, P. Mailley, M. A. Pinault, F. Jomard, and P. Bergonzo, High reactivity and stability of diamond electrodes: The influence of the B-doping concentration, Phys Status Solidi A 206 (9), 2063-2069 (2009)

[7] P. Vitorge. Chimie des actinides. Techniques de l'ingnieur. BN 3520-2 (1999).

[8] N.A Talvitie. Electrodeposition of actinides for a spectrometric determination. Analytical Chemistry, Vol 44, N2 (1972) 280283.

[9] A. Becerril-Vilchis, A. Corts, F. Dayras, J. de Sanoit. A method for the preparation of very thin and uniform $\alpha$-radioactive sources. Nuclear Instruments and Methods in Physics Research A 369 (1996) 613-616.

[10] V. Tsoupko-Sitnikov, F. Dayras, J. de Sanoit, D. Filossofov. Application of rotating disk electrode technique for the preparation of $\mathrm{Np}, \mathrm{Pu}$ and Am $\alpha$-sources, Applied. Radiation and Isotopes. Vol 52, N 3, (2000) 357 364.

[11] M. Ciobanu, N. Herrmann, K. D. Hildenbrand, T. I. Kang, M. Ki, and A. Schttauf, A charge sensitive amplifier for time and energy measurements, Proc. IEEE Nucl. Sci. Symp., Dresden, Germany, 2008, vol. N30-20, pp. 2028-2032.

[12] SRIM - The Stopping and Range of Ions in Matter, James F. Ziegler, http://www.srim.org

[13] Ryszard Broda, Philippe Cassette and Karsten Kossert, Radionuclide metrology using liquid scintillation counting, Metrologia 44:4, S36-S52, 2007

[14] Q.T. Tran, J. de Sanoit, M. Pomorski, C. Mer-Calfati, P. Bergonzo Optimization of the efficiency of Diamond Based Alpha Spectrometers in Solutions, this proceedings no. 1162

[15] J. de Sanoit, M. Pomorski et C. Mer.,Détecteur alpha électrochimiquement assisté pour la mesure nucléaire en milieu liquide et procédé associé. French patent n 1058150 du 7 octobre 2010 